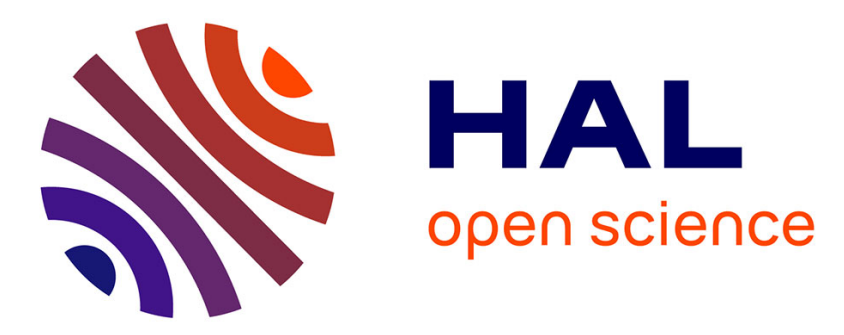

\title{
La dette publique et la reconfiguration des relations entre les financiers et l'État durant la dernière décennie de l'Ancien Régime espagnol (1823-1834)
}

Jean-Philippe Luis

\section{- To cite this version: \\ Jean-Philippe Luis. La dette publique et la reconfiguration des relations entre les financiers et l'État durant la dernière décennie de l'Ancien Régime espagnol (1823-1834). Anne DUBET, Jean Philippe LUIS. Les financiers et la construction de l'État France, Espagne (XVIIe-XIXe siècle), Presses Uni- versitaires de Rennes, pp.155-174, 2011, 978-2-7535-1699-1. hal-01318827}

\section{HAL Id: hal-01318827 \\ https://hal.uca.fr/hal-01318827}

Submitted on 20 May 2016

HAL is a multi-disciplinary open access archive for the deposit and dissemination of scientific research documents, whether they are published or not. The documents may come from teaching and research institutions in France or abroad, or from public or private research centers.
L'archive ouverte pluridisciplinaire HAL, est destinée au dépôt et à la diffusion de documents scientifiques de niveau recherche, publiés ou non, émanant des établissements d'enseignement et de recherche français ou étrangers, des laboratoires publics ou privés. 


\title{
La dette publique et la reconfiguration des relations entre les financiers et l'État durant la dernière décennie de l'Ancien Régime espagnol (1823-1834)
}

\author{
Jean-Philippe Luis
}

En 1831, une caricature élaborée par les milieux ultras opposés à Ferdinand VII circulait sous le manteau à Madrid. On y voyait «l'Espagne comme un squelette, qui n'a de chair que sur un mollet : ici accrochés Aguado, Burgos, Ballesteros, etc. (...) Encima avec une pierre tombale où était inscrit Encima Piedra $»^{1}$. Les hommes présentés ici comme responsables de l'agonie de l'Espagne sont un banquier (Alejandro María Aguado), un journaliste ancien haut fonctionnaire reconverti en partie dans les affaires (Javier de Burgos), le ministre des finances (Luis López Ballesteros) et l'un de ses proches collaborateurs, Víctor Encima y Piedra qui était alors directeur de la Caisse royale d'amortissement de la dette. La même perception existait dans d'autres secteurs politiques. À la même période, Sainz de Andino, un ancien afrancesado devenu membre du Conseil des Finances s'insurgeait : "Comment peut-on voir sans indignation que les mêmes chefs qui devraient donner l'exemple de la pureté, de l'intégrité et du désintérêt dans la perception et la distribution des recettes des Finances Royales, ont des participations dans l'affermage de celles-ci et dans les contrats des biens en monopoles $»^{2}$. La principale personne visée derrière ces propos était Gaspar de Remisa, un banquier catalan détenteur d'une des plus grosses fortunes du pays qui était devenu directeur du Trésor Royal. Ces deux exemples indiquent l'existence d'une perception répandue de collusion entre la direction de l'administration des finances et les milieux d'affaires. L'existence d'une telle dénonciation laisse supposer qu'il s'agit d'une nouveauté. Or, tous les travaux sur le XVIIIe siècle, ceux menés en particulier par plusieurs auteurs de ce livre, ont prouvé le contraire. Quelle réalité recouvre donc cette collusion sous Ferdinand VII et en quoi est-elle nouvelle par rapport aux pratiques antérieures ?

Ce terrain est encore très largement à défricher. Si des travaux, pour certains déjà anciens, existent sur les finances de l'État ${ }^{3}$, rien n'a été fait sur les financiers, alors qu'ils ont été l'objet d'études en amont, sous Charles IV, et en aval, sous Isabelle $\mathrm{II}^{4}$. On ignore en particulier presque tout sur le détail de l'interface entre État et financiers, à savoir la question de la régie indirecte, c'est-à-dire celle des contrats de droit privé passés entre des entreprises et l'État pour la fourniture de biens ou de services. À l'exception du secteur minier, il n'existe aucune étude comparable à celles

\footnotetext{
${ }^{1}$ Arias Teijeiro : 1967-1968, t. 3, 24 février 1831, p. 12

${ }^{2}$ Cité dans : Suárez : 1970a, p. 459.

${ }^{3}$ Fontana : 2001 ; Artola : 1986 ; Broder : 1981.

${ }^{4}$ Par exemple pour le XVIIIe siècle Aquerreta : 2001 et Torres Sánchez : 2000. Pour le règne d'Isabelle II, Otazu : 1987.
} 
qui se sont penchées sur les différentes facettes de la régie indirecte (contrats d'affermage, contrats d'asiento pour l'approvisionnement aux armées, concessions...) aux XVIIe et XVIIIe siècles ${ }^{5}$. Les raisons d'une telle situation sont d'abord liées à un désintérêt pendant longtemps très marqué pour une période de sinistre mémoire que l'historiographie libérale espagnole a désignée sous le nom de "décennie abominable $»^{6}$. La dernière restauration de l'absolutisme en 1823, avec l'aide des troupes françaises des Cent mille fils de Saint-Louis, a été en effet une phase de répression et d'étouffement de la vie politique, intellectuelle et artistique. L'autre raison est liée à la dispersion et à la disparition de nombreuses sources, dans cette période charnière de refonte complète des structures administratives qui caractérise les années 1808-1840.

Je vais tenter ici de décrypter certains des aspects des relations entre État et financiers au travers d'un exemple, celui des emprunts massifs que l'Espagne a émis sous la forme d'obligations à la Bourse de Paris, puis à celles d'Amsterdam et d'Anvers entre 1824 et 1832 . Mon hypothèse est que cet épisode joue un rôle essentiel dans l'émergence d'une nouvelle configuration des relations entre le roi, l'appareil administratif des finances et les financiers, à la faveur d'une conjoncture politique et économique européenne et américaine nouvelle.

\section{L'affaire des emprunts}

\subsection{Un pays étranglé par la dette}

Telle était en effet la situation de la monarchie espagnole en 1823. L'endettement des années 1820 était le fruit d'une série d'emprunts intérieurs et, à partir de 1768, d'emprunts extérieurs sur les places boursières européennes. Le fardeau s'était alourdi dans les années 1780 avec la création de bons du trésor (vales reales) destinés au financement de la guerre contre l'Angleterre. Le procédé fut utilisé à nouveau pour financer les besoins des guerres de la Révolution et de l'Empire, ce qui eut pour effet de faire chuter la valeur de ces bons qui, en 1807, ne valaient plus que $57 \%$ de leur valeur nominale. La France napoléonienne imposait une contribution à l'Espagne, comme à tous ses alliés ou satellites. Dans l'impossibilité d'y faire face, le gouvernement recourut pour la première fois à un financier aventurier en la personne d'Ouvrard. En 1804, ce dernier fut chargé par le roi de contracter un emprunt en Hollande. En 1808, la dette publique intérieure et extérieure s'élevait déjà à dix années de revenus.

Avec la Guerre d'Indépendance et la perte des colonies américaines, les recettes fiscales s'effondrèrent. L'absolutisme restauré en 1814 hérita d'un pays ruiné et d'une dette publique que la direction générale du crédit public évaluait à plus de 11000 millions de réaux (2933 millions de francs). Les intérêts annuels s'élevaient alors à 212,5 millions de réaux ${ }^{7}$, à une époque où les recettes annuelles de l'État atteignaient en moyenne 658 millions de réaux ${ }^{8}$. La gestion financière calamiteuse de la première restauration et la crise européenne de 1819 ne firent qu'accentuer les déficits. En 1820, la seule dette intérieure de l'État s'élevait à 13000 millions de

\footnotetext{
${ }^{5}$ Sur le secteur minier : Chastagnaret : 1993 et 2000. J'ai effectué une comparaison entre cinq gros négociants-financiers sous le règne de Ferdinand VII dans Luis : 2011. Pour la définition de 1'asiento, je renvoie à la définition donnée dans Dubet coord. : 2008, p. 307-308.

${ }^{6}$ Cette situation est heureusement moins marquée depuis la fin des années 1990. Voir le numéro spécial de la revue Ayer: El reinado de Fernando VII y su imagen.

Artola : 1973, p. 296.

${ }^{8}$ Fontana : 1971, p. 69.
} 
réaux, soit probablement $160 \%$ du revenu national de $1800.45 \%$ de cette dette avait été générée après $1808^{9}$. Le nouveau régime issu de la révolution libérale de 1820 ne résolut rien et accentua le poids de la dette extérieure : il fut contraint de reconnaître la dette de Hollande qui s'élevait alors à 543,7 millions de réaux (capital plus intérêts impayés) ${ }^{10}$ pour pouvoir emprunter. Ce que l'on appelle l'emprunt des Cortes, c'està-dire de la période 1820-1823, correspond en réalité à plusieurs emprunts, contractés en France et en Angleterre.

Au début de la seconde restauration (1823), les plus lucides des responsables politiques avaient fini par accepter l'idée que la perte des ressources fiscales de l'Empire, l'appauvrissement du pays et la crise déflationniste qui l'affectait rendaient illusoire tout accroissement déterminant des ressources fiscales. La dégradation des finances publiques était donc structurelle et ne permettait pas une amélioration à court ou à moyen terme. Chaque année accentuait le déficit, d'un montant évalué en 1824 par l'ambassadeur de France entre 200 et 300 millions de réaux ${ }^{11}$.

La nouvelle politique élaborée par le ministre des finances, Luis López Ballesteros à partir de 1824 correspond à une tentative d'adaptation dans des limites politiques très étroites. En effet, l'objectif ultime était de sauver l'absolutisme. Les dépenses publiques étant devenues depuis longtemps trop élevées pour un État qui avait perdu la manne fiscale de l'Empire colonial et du monopole qui y était intimement associé, López Ballesteros mit en place une politique de réduction drastique des dépenses et d'augmentation de la pression fiscale. Ce projet était dangereux politiquement car il était porteur d'un fort mécontentement populaire, que les libéraux en exil et les ultras à l'intérieur escomptaient récupérer à leur profit. Par prudence, les réformes furent étalées en espérant qu'elles seraient ainsi mieux acceptées. Dans l'intervalle, il était nécessaire de recourir à l'emprunt pour combler l'écart considérable entre recettes et dépenses et faire face aux remboursements de la dette.

Vivre à crédit ne pouvait se faire en ayant recours aux emprunts sur le marché intérieur. Les appels aux consulats des villes marchandes avaient été un échec entre 1814 et 1820 . L'emprunt de 1818 pour financer expédition en Amérique n'avait été couvert qu'à $50 \%$, malgré des taux de $8 \%$ offerts $^{12}$. L'appauvrissement du pays avait réduit les possibilités de financement intérieur, et les détenteurs de capitaux ne faisaient guère confiance à un État mauvais payeur. Cette attitude n'était pas nouvelle. Dès le dernier tiers du XVIIIe siècle, l'État s'avérait incapable de créer un marché dynamique de la dette publique en entretenant l'incertitude et l'arbitraire à l'égard des créanciers potentiels ${ }^{13}$. Cette réalité était accentuée après la guerre d'Indépendance par un phénomène non quantifié de fuite des capitaux vers les titres de la dette des grands États du Nord de l'Europe, surtout français et anglais, réputés beaucoup plus sûrs ${ }^{14}$. L'argent frais ne pouvait donc être trouvé qu'à l'extérieur. Mais là encore, les possibilités étaient très limitées. Le gouvernement absolutiste restauré en 1823 avait refusé de reconnaître la dette émise par le régime libéral, dont les titres avaient été émis et achetés avant tout à Londres, Amsterdam et Anvers. Ce refus, perçu par la

\footnotetext{
${ }^{9}$ Tedde de Lorca : 2000, p. 3.

${ }^{10}$ Fontana : 2001, p. 156.

11400 millions de recettes pour 600 à 700 millions de dépenses. Broder : 1981, p. 422.

${ }^{12}$ Artola : 1986, p. 80.

${ }_{13}$ Torres Sánchez : $2008 \mathrm{c}$.

${ }^{14}$ Ceci est la donnée principale qui ressort de la consultation des archives des consulats de France à Madrid et Cadix. Centre des Archives Diplomatiques de Nantes. Actes notariés postes, Consulat de France à Madrid, registre $n^{0} 1$ à 5 ; actes notariés postes, Consulat de France à Cadix, tomes 46 à 48 .
} 
haute banque européenne comme une erreur grossière, fermait de fait les bourses de ces pays à un futur emprunt espagnol. Seule restait la place parisienne.

La bourse de Paris était alors en plein essor, stimulée par le déplacement des capitaux de l'Europe rhénane vers l'ouest à la faveur de la fin des guerres napoléoniennes, ainsi que par le lancement des emprunts d'État français ${ }^{15}$. La ville connaissait de plus une intense activité immobilière spéculative. Ce dynamisme financier et boursier apparaissait aussi dans le succès public des valeurs boursières. On évalue en 1830 à 189000 le nombre de détenteurs de titres, succès qui se reflète dans la vogue des manuels d'usage des placements à la Bourse, véritable manuels du parfait spéculateur qui décrivent en particulier dans le détail toutes les subtilités des placements à terme ${ }^{16}$.

Par ailleurs, le gouvernement français avait intérêt à ce que l'Espagne trouve des fonds. En effet, l'expédition des Cent mille fils de Saint-Louis venant de rétablir l'absolutisme avait été coûteuse et Ferdinand VII réclamait le maintien provisoire de garnisons françaises pour assurer la sécurité de son trône. Ces coûts dénoncés par l'opposition libérale devaient être en partie comblés par une indemnité versée par l'Espagne $^{17}$. La France avait donc tout intérêt à ce que l'Espagne trouve des fonds. C'est probablement cette nécessité qui poussa Villèle à autoriser la cotation des titres d'États étrangers à la Bourse de Paris, le 12 novembre 1823.

Le dynamisme de la place boursière parisienne n'était cependant pas suffisant pour garantir le succès d'un emprunt d'État espagnol. En effet, la réputation internationale justifiée de mauvais payeur et celle d'incurie administrative et financière de l'Espagne explique qu'aucune grande banque n'ait accepté de prendre à sa charge cet emprunt. Un tel projet était perçu comme trop risqué et peu attractif devant la concurrence forte des grands États emprunteurs (la France, l'Angleterre, l'Autriche), réputés meilleurs payeurs. Des négociations avaient été entamées en 1823 avec Rothschild, Outrequin et Jauge, avec Baguenault et Seillères, puis en 1825 auprès de l'ensemble de la haute banque parisienne. Elles n'aboutirent pas, faute de garanties suffisantes, faute d'engagement du gouvernement français à garantir l'emprunt, et d'assurances concernant la reconnaissance de la dette du Trienio liberal $^{18}$. Le gouvernement espagnol n'avait donc d'autre recours que de se tourner vers des banquiers de second ordre dans un contexte boursier hautement spéculatif.

\subsection{La mise en place des emprunts}

Pour parer au plus pressé, et avant même la victoire définitive des troupes françaises, un accord fut passé par le gouvernement provisoire auprès de la maison Guebhard de Paris en septembre 1823. Il s'agissait d'un emprunt, appelé Emprunt Royal, de 334 millions de réaux nominal, soit d'environ 90 millions de francs, pris à $60 \%$ et répartis en 83500 obligations. Guebhard était un banquier de second ordre et fut incapable de remplir ses obligations. C'est alors qu'entra en scène Alexandre Marie Aguado. Cet homme encore jeune, il avait 38 ans, était un ancien officier afrancesado qui, en exil à Paris, avait déjà bâti une affaire prospère de commerce du vin et de produits espagnols et américains. Il faisait aussi de la gestion de titres pour

\footnotetext{
${ }_{16}^{15}$ Levy-Leboyer : 1964, p. 440.

${ }^{16}$ Par exemple Jacques Bresson, Des fonds publics en France et des opérations de la Bourse de Paris, Paris, Bachelier, 1820. Voir Gontard : 2000, p. 244-250.

${ }^{17}$ Le coût réel de l'opération était évalué en 1825 à 170 millions de francs et le gouvernement espagnol reconnaissait en janvier 1824 une dette de 34 millions de francs. Butrón Prida : 1996, p. 4574. Je renvoie aussi sur cette question à l'article de Sébastien Kott que l'on trouvera dans ce livre.

${ }^{18}$ Luis : 2009, p. 146-147 et p. 160-162.
} 
ses concitoyens à la Bourse de Paris, et était lié au négociant-banquier Guérin de Foncin au travers d'activités commerciales à Rouen. Aguado faisait partie de la cohorte des petits escompteurs parisiens et avait déjà acquis une certaine réputation car il pouvait en 1823 escompter des lettres auprès de la Banque de France. Il restait toutefois perçu avant tout comme un commerçant habile dans le maniement des effets de commerce, et, pour la police, comme « un courtier marron ${ }^{19}$.

Aguado s'était vu confier le placement à la bourse d'un certain nombre d'obligations de l'emprunt Guebhard, par l'intermédiaire du représentant spécial du gouvernement espagnol pour l'emprunt, Joaquín Carrese ${ }^{20}$. Les deux hommes s'entendirent pour écarter Guebhard en mars 1824, Aguado finissant par reprendre à son compte le placement de l'emprunt à partir de septembre. Cet emprunt s'avérait cependant insuffisant et le gouvernement espagnol tenta de lever un nouvel emprunt de 800 millions de réaux (environ 200 millions de francs). De nombreux projets furent présentés. Ils échouèrent tous, y compris celui que tenta d'imposer le nouveau chef du gouvernement espagnol, Céa Bermúdez, qui réunit à Paris le 6 août 1825 une conférence avec des représentants de la Sainte-Alliance afin de convaincre Rothschild d'accepter cet emprunt ${ }^{21}$. Dans l'impasse, le gouvernement espagnol dut se tourner vers Aguado ce que confessa plus tard le ministre des Finances Luis López Ballesteros : « le génie entreprenant et audacieux du banquier Alejandro Aguado était le seul qui pouvait prêter quelques secours à l'Espagne (... ), on ne peut pas dire que ce qu'il proposait était le plus avantageux, mais c'était le plus rationnel, de plus personne d'autre ne nous aurait aidé ${ }^{22}$. Le soutien du gouvernement à Aguado se renforça quand le banquier accepta en 1826 de mener à bien la vaste fraude de la conversion de l'Emprunt Royal qui, sous couvert d'une conversion, devint émission d'un nouvel emprunt.

Aguado devint désormais banquier de la Cour d'Espagne et plaça à ce titre cinq emprunts d'État espagnols de 1824 à 1831, pour une valeur totale de 540 millions de francs (plus de deux milliards de réaux). Sa réussite à Paris lui ouvrit la porte des bourses d'Anvers et d'Amsterdam où il réussit à lancer en janvier 1830 un emprunt de près de 90 millions de francs ${ }^{23}$.

\section{Derrière l'emprunt, un réseau}

Cette affaire des emprunts est au cœur de la problématique politique de la seconde restauration. L'analyse de la chaîne des acteurs des emprunts permet de faire émerger l'existence d'un groupe de financiers et de membres de la haute administration intimement liés à la politique menée par le ministère des Finances.

\subsection{Un réseau complexe}

L'homme à l'origine du premier emprunt est Juan Bautista Erro y Aspiroz ${ }^{24}$. Cet ingénieur des mines était passé aux finances avant la guerre d'Indépendance en devenant intendant. Antilibéral affirmé, il devint ministre des finances du gouvernement provisoire en juillet 1823. C'est à ce titre qu'il envoya à Paris un de ses parents $^{25}$, Joaquín Carrese, comme représentant du gouvernement auprès du banquier

19 AN, F7, 12000, rapport du 18 décembre 1824. Des détails dans Luis : 2009, p. 126-144.

${ }^{20} \mathrm{~J}$ 'ai exposé toute cette affaire dans Luis : 2009, chap. 5.

${ }^{21}$ Broder : 1981, p. 430-431 et Gille : 1965, p. 131-132.

${ }^{22}$ Archivo del Ministerio de Hacienda (Madrid), Fondo López Ballesteros, 5/4.

${ }^{23}$ Broder : 1981, p. 443.

${ }^{24}$ Casquero Fernández, Jaramillo Guerreira : 1995.

${ }^{25} \mathrm{La}$ famille Erro était correspondante des frères Carrese lorsque ceux-ci étaient étudiants au 
Guebhard pour veiller au bon déroulement du placement de l'Emprunt Royal à la bourse : remettre progressivement les obligations, surveiller les cours des valeurs et veiller à l'envoi de fonds en Espagne. Dans le cadre de ses fonctions, Carrese rencontra Aguado à Paris et les deux hommes devinrent des proches. Aguado encaissait les lettres sur la Banque de France que Carrese percevait comme commission . L'un des détracteurs d'Aguado le décrit comme un «ami, conseiller et directeur » de Carrese ${ }^{27}$. Les deux familles se connaissaient certainement car Joaquín Carrese était étudiant au séminaire de Vergara en même temps qu'un frère et que deux cousins d'Alexandre Marie Aguado. Par ailleurs, Aguado fut peut-être recommandé à Carrese par Pedro de Grimarest, un des dirigeants de l'insurrection royaliste de 1823, proche de Erro. Grimarest était un ancien ami d'Aguado. Ils s'étaient probablement connus au début de la guerre d'Indépendance. En effet, Grimarest exerçait alors des fonctions importantes dans l'armée du centre dans laquelle Aguado avait combattu avant de changer de camp au printemps 1810. Les deux hommes correspondaient au début des années 1820 puis devinrent beaux-frères en 1826 quand Grimarest épousa une sœur d'Alexandre Marie Aguado .

L'échec de l'emprunt, le changement de ministre des finances et la désinvolture de Carrese (d'après la police française, il s'occupait « avec insouciance des emprunts, sauf si cela lui rapporte $»{ }^{30}$ ) déclenchèrent son remplacement par un autre commissaire du gouvernement, Javier de Burgos. Ce dernier était une vieille connaissance d'Aguado. Ils avaient connu ensemble la retraite d'Andalousie, à partir d'août 1812 car Burgos avait été lui aussi afrancesado (il fut sous-préfet sous Joseph Bonaparte). Journaliste et écrivain, Burgos se présente aussi comme un réformateur et comme un des acteurs de la politique mise en place par le ministre des Finances Luis López Ballesteros ${ }^{31}$. À Paris, Burgos était très lié à l'ambassadeur intérimaire d'Espagne, le marquis de Casa Irujo, fils du chef du gouvernement espagnol mort en janvier 1824. Servait aussi à l'ambassade le célèbre Sebastián de Miñano, un autre afrancesado, vieille connaissance d'Aguado à Séville. Ces hommes avaient des relations dans le monde politique parisien car ils avaient servi d'informateurs et de médiateurs à Martignac en 1823 lors de l'invasion de la péninsule par l'armée des Cent mille fils de Saint-Louis ${ }^{32}$. À Madrid, outre le ministère des finances, le groupe trouvait un relais auprès du comte d'Ofalia, ministre durant six mois en 1824, puis ambassadeur à Paris en 1829. Ofalia et le marquis de Casa Irujo étaient par ailleurs membres d'institutions commerciales et financières patronnées par l'État: tous deux appartenaient à la direction de la Banque Saint-Charles et Ofalia avait intégré l'organe directeur de la Compagnie Royale des Philippines au titre des actionnaires. Casa Irujo devint plus tard, de 1831 à 1833, l'un des trois membres de la direction de la nouvelle banque de San Fernando . Au ministère des finances, l'un de leurs relais

séminaire de Vergara dans les années 1790. Chaparro Saínz : 2009, p. 345.

${ }^{26}$ AHN, Hacienda, legajo $737^{1}$. «Informe para el señor Don L. Guebhard contra la acción del señor Aguado ».

Suárez : 1970b, vol. 2, p. 36.

${ }^{28}$ Chaparro Saínz : 2009, p. 589-590.

${ }^{29}$ Luis : 2009, p. 193.

${ }^{30}$ AN, F7, 11994, rapport du 12 mai 1824.

${ }^{31}$ Une rapide biographie dans Gil Novales : 1991.

${ }^{32}$ Luis : 2009, p. 210-211.

${ }^{33}$ Cet établissement fut créé en 1782 et avait pour finalité première l'échange des bons du trésor (vales reales). Il fut remplacé en 1829 par la Banque de Saint-Ferdinand. Tedde de Lorca : 1988 et 1999.

${ }^{34}$ Molas Ribalta : 2008, p. 273 ; Francisco Olmos : 1997, p. 342-347. 
principaux était Juan Ignacio Vincenti, un homme que l'on connaît mal, qui avait été membre du Conseil des Finances et qui était devenu le directeur de la Caisse royale d'amortissement de la dette créée en février 1824. C'est Vincenti qui proposa que Javier de Burgos soit désigné comme successeur de Carrese à Paris pour la gestion des emprunts . Il fut remplacé à sa mort, en 1826, par Esteban de Goicorrotea qui était auparavant trésorier de la Caisse royale d'amortissement. On ignore tout de ce personnage à l'exception des affaires commerciales qu'il faisait avec une maison de Bayonne $^{36}$. À sa mort, en 1829, la direction de la Caisse d'amortissement passa à Victoriano Encima y Piedra, membre de la commission sur le commerce des Indes, puis à partir de 1825 d'une junte chargée des «privilèges de la Compagnie des Philippines » où il croisait Ofalia ${ }^{37}$. Le lien direct ou indirect entre Encima y Piedra, Aguado et Carrese est peut-être ancien, car le premier avait été lui aussi étudiant au séminaire de Vergara .

Dans un premier temps, le «patron » de ce groupe était Ugarte, favori du roi jusqu'en 1824. Ce patronage est révélé par la conspiration fomentée par le comte de La Puebla et par Casa Irujo, aidés de Burgos, pour obtenir le renvoi du nouveau chef du gouvernement, Francisco Céa Bermúdez, et le rappel d'Ugarte ${ }^{39}$. La logique de ce groupe est purement de réseau. Il a une forte coloration andalouse ou navarroandalouse. Aguado, Ofalia, Casa Irujo et Burgos appartenaient aux oligarchies urbaines andalouses issues du commerce. Encima y Piedra appartenait au monde du grand commerce gaditain dans lequel une branche de la famille Aguado demeurait. Erro et Carrese étaient les seuls vrais navarrais, mais Aguado, Casa Irujo, Encima y Piedra et Miñano étaient d'origine navarraise et le séminaire de Vergara jouait un rôle fondamental de lien entre cette région et les Navarrais d'origine qui avaient intégré les élites andalouses par le commerce. Carrese, Encima y Piedra, Erro et les frères d'Aguado y avaient été étudiants ou y étaient liés. Ce réseau pourrait donc être un avatar tardif de la célèbre « hora navarra » décrite par Caro Baroja ${ }^{40}$.

Le groupe que l'on vient de décrire n'est pas politique car les hommes en question sont des ultras royalistes (Erro, Grimarest, Ugarte) et des anciens afrancesados dont certains ont flirté un temps avec le libéralisme modéré (Burgos, Miñano). Il s'agit là d'une action politique typique d'Ancien Régime, c'est-à-dire, d'une période durant laquelle faire de la politique signifie être intégré à l'un des réseaux concurrents qui luttent pour obtenir la faveur du roi. Avec la mise à l'écart durable d'Ugarte, le groupe trouve un nouveau « patron » en la personne du ministre des finances López Ballesteros. Il acquiert par là même une plus grande cohérence idéologique en devenant le maître d'œuvre de la politique réformiste que le ministre parvient à faire accepter au roi jusqu'en 1832, c'est-à-dire une politique de réforme administrative et financière dont l'objectif est de sauver la monarchie absolue. Ce réseau est en concurrence avec d'autres réseaux, en particulier, celui dirigé à Madrid par Regato, un ancien révolutionnaire passé à l'ultracisme qui était devenu directeur de la police privée du roi. Regato s'appuyait sur Calomarde, ministre de Grâce et Justice, et avait comme relais à Paris le banquier Haber et surtout le puissant

${ }^{35}$ Burgos : 1850, p. 118.

${ }^{36}$ Suárez : 1970b, vol. 1, p. 239.

${ }^{37}$ AHN, Hacienda, legajo 2665, exp. 48.

${ }^{38}$ Chaparro Saínz : 2009, p. 595.

${ }_{40}^{39}$ Luis : 2009, p. 196-200.

${ }^{40}$ Il ne s'agit ici que d'un réseau parmi les multiples réseaux liés à la Navarre qui existaient au XVIIIe siècle. Les travaux récents ont montré que ces réseaux étaient souvent concurrents. Torres Sánchez: 2010a, p. 27. Caro Baroja évoque entre autres la figure d'Aguado pour illustrer ce qu'il nomme l'époque de la « liquidation » de « la hora navarra » (Caro Baroja : 1969, p. 396). 
négociant valencien Vicente Bertrán de Lis, grande figure du libéralisme exilé à Paris, qui ambitionnait pourtant de remplacer Aguado comme banquier du roi $^{41}$ et que Regato recommandait pour les contrats de fourniture aux armées ${ }^{42}$.

\subsection{Une instrumentalisation de l'État ?}

Tous les membres du groupe Aguado ont connu un enrichissement important à la faveur des emprunts émis entre 1824 et 1832. Cet objectif est clair dès le départ: Erro et Carrese ont passé en 1823 un accord secret avec Guebhard afin que ce dernier leur reverse $50 \%$ de la commission qu'il devait percevoir dans le placement de l'emprunt ${ }^{43}$. Cet accord était probablement le prix que Guebhard avait consenti à payer à Erro pour que l'emprunt lui fût confié. Carrese s'enrichit très rapidement en quelques mois. La police française estimait qu'il s'était constitué un capital de 800000 francs en juin 1824, puis de 2 millions en novembre ${ }^{44}$. Aguado fut bien sûr le principal bénéficiaire. Il devint en moins de dix ans l'une des dix personnes les plus fortunées de France. Il laissait à sa mort plus de vingt millions de francs (plus de cent millions de réaux). L'origine de cette fortune se trouve bien sûr dans les commissions perçues, évaluées par le ministère des finances espagnol à 28 millions de francs en neuf ans, de 1824 à $1833^{45}$. À ceci s'étaient ajoutées les sommes probablement colossales acquises par la spéculation. C'est par cette voie qu'il permit aux autres membres du réseau à Paris de s'enrichir. Burgos, Casa Irujo, Ofalia, Miñano à un moindre degré, en furent les principaux bénéficiaires. Burgos ne possédait presque rien en arrivant à Paris ${ }^{46}$ et la fortune qu'il acquit grâce aux emprunts était si visible qu'il fut mis en accusation en 1834 par la chambre du nouveau régime libéral. Il se défendit sans masquer le rôle de la spéculation : "par ces moyens nobles, le célèbre Rothschild construisit et consolida son immense fortune, dont l'acquisition rapide et honnête n'est remise en cause par personne ${ }^{47}$. Aguado arracha le lancement d'un dernier emprunt en 1831 en ayant versé des commissions occultes au directeur de la Caisse d'amortissement, Encima y Piedra, et même au roi à qui il aurait offert 100000 francs en titres de la dette ${ }^{48}$.

Profiter du service de l'État pour s'enrichir n'avait rien d'exceptionnel ${ }^{49}$. La nouveauté vient de l'ampleur de l'enrichissement. Il laisse penser à une instrumentalisation de l'État par un groupe de prédateurs et à une collusion entre financiers et hauts responsables politiques et administratifs. Cette dernière impression est confortée par les relations étroites entre les membres du groupe appartenant à la haute administration et le monde du négoce et de la banque. Ofalia et Casa Irujo, comme on l'a déjà souligné, étaient membres de la direction de la Banque de SaintCharles et Ofalia était actionnaire et l'un des dirigeants de la Compagnie Royale des Philippines. Par ailleurs, le père de Casa Irujo, tout en étant diplomate, avait fait des affaires commerciales à Cadix après la guerre d'Indépendance ${ }^{50}$. Encima y Piedra a

\footnotetext{
${ }^{41}$ Sánchez Mantero : 1975, p. 92.

${ }^{42}$ Morange : 1977 , p. 509.

43 AHN, Hacienda, legajo $737^{1}$. L'affaire est révélée par les arguments développés par Guebhard dans le procès que lui intente Aguado en juillet 1824: «Informe para el señor Don L. Guebhard contra la acción del señor Aguado ».

AN, F7 11994, rapports du 7 juin et du 11 novembre 1824.

${ }^{45}$ Luis : 2009, p. 182.

${ }_{47}^{46}$ AN, F7 12032, dossier 977e.

${ }_{47}^{47}$ Burgos : 1850, p. 120.

${ }^{48}$ Arias Teijeiro : 1967-1968, t. 3, p. 74, 21 mars 1831.

${ }^{49}$ Voir l'entrée « corruption » rédigée par Anne Dubet dans Legay dir. : 2010.

${ }^{50}$ Ozanam : 1998, p. 343.
} 
un profil plus proche de ce que l'on pouvait rencontrer dans la deuxième moitié du XVIIIe siècle : il s'agit d'un négociant de Cadix, représentant à Madrid de la Junta de diputados de comercio de la ville de Cadix en 1816, devenu haut fonctionnaire des Finances après avoir assumé des tâches dans différentes commissions liées au ministère entre 1819 et $1829^{51}$.

Par ailleurs, l'action du ministère des finances a favorisé l'impression d'instrumentalisation. Il a accepté qu'Aguado place une grande partie des actions de l'Emprunt Royal en 1824 et 1825 sans effectuer d'avance. Aguado a ainsi spéculé avec de l'argent qui ne lui appartenait pas. Le ministère fut surtout à l'origine d'une fraude massive. En effet, c'est dans le plus grand secret, en conseil des ministres, qu'il est prévu d'émettre un nouvel emprunt à la Bourse de Paris sous couvert d'une conversion de l'emprunt Guebhard en rente perpétuelle à 5\%. La conversion ne touche qu'un million de réaux sur les 150 millions visés, mais Aguado en profite pour émettre 565 millions de cette rente perpétuelle. Cette opération était rigoureusement interdite par les autorités boursières et politiques françaises, mais nombreux furent les agents de change et les banquiers parisiens qui profitèrent de cette manne spéculative jusqu'à ce que le scandale éclate en janvier $1829^{52}$. Les manœuvres frauduleuses furent donc subies ou encouragées par le pouvoir politique. De manière très lucide et cynique, Aguado écrivait en 1828 à López Ballesteros : " Afin d'obtenir notre crédit, j'ai dû ne pas omettre les vérités absolues, les vérités conditionnelles, des exagérations évidentes et des mensonges qualifiés ; en somme, je me suis identifié au gouvernement » .

L'impression de collusion et d'instrumentalisation des finances de l'État par un groupe doit cependant être nuancée. Tout d'abord, l'État absolutiste est contraint à la fraude parce qu'il y va de sa survie : seul ce moyen permet de trouver de l'argent frais. Ensuite, l'équilibre entre financiers et administration évolue dans le temps. La phase qui va de 1824 à 1829 correspond à celle des enrichissements personnels les plus rapides et les plus forts, sans que l'on perçoive de réels mécanismes de contrôle financier. Les caisses de l'État sont vides et on ferme les yeux sur les moyens employés pour les remplir. Les émissaires du gouvernement à Paris et Aguado semblent avoir les mains libres dans la mesure où ils parviennent à placer les emprunts en mettant en œuvre la fraude de 1826 et où ils effectuent les versements prévus à la Trésorerie générale. Le contrôle sur le banquier s'accroît toutefois à partir de 1829. Le scandale de la fausse conversion et une situation budgétaire intérieure qui s'améliore un peu conduisent l'administration des finances à se pencher sérieusement sur les manœuvres d'Aguado. Cette évolution va de pair avec un fort accroissement du personnel affecté à la Caisse royale d'amortissement. Les bureaux de la Caisse n'abritaient que cinq personnes lors de sa création en 1824. Six ans plus tard, ils étaient 146, puis 197 en $1833^{54}$. Les moyens humains de contrôle s'accroissaient et avec eux la pression sur Aguado. Des rapports négatifs s'accumulaient sur sa gestion. Il démissionna de sa fonction en 1832, en conflit avec l'administration des finances qui lui réclamait 4 millions de francs, alors que le banquier revendiquait 7 millions de francs pour solder les comptes de son action comme banquier de la cour. Le contentieux ne fut jamais réglé. Le gouvernement espagnol poursuivit les héritiers d'Aguado après la mort de ce dernier en 1842. La procédure ne s'arrêta qu'en 1866 pour cause de prescription.

\footnotetext{
${ }^{51}$ AHN, hacienda, legajo 2665, exp. 48.

${ }^{52}$ Luis : 2009, p. 165-174.

${ }^{53}$ AHN, Hacienda, legajo $736^{2}$, lettre du 23 février 1828

${ }^{54}$ Guía de forasteros de Madrid, Madrid, Imprenta real, 1824, 1830 et 1833.
} 
Ainsi, le retour d'une capacité de contrôle par l'administration des finances montre qu'on ne peut limiter les enrichissements faramineux réalisés à la faveur des emprunts à une instrumentalisation pure et simple d'un clan financiaro-administratif. L'existence même de ce contrôle prouve que l'objectif ultime reste de remplir les caisses de l'État, un État qui n'est pas un écran de fumée. Tous les rapports émis autour de cet emprunt, par exemple ceux de Pedro Sainz de Andino ou d'Antonio Martini $^{53}$, renvoient à la notion de service de l'État, une notion qui n'a pas disparu et qui reprend les termes employés à l'époque des Lumières. En système absolutiste, le service de l'État signifie fournir au roi les subsides qui permettront d'appliquer la politique qu'il souhaite mettre en œuvre. Le détail des moyens utilisés à cet effet importe peu et l'enrichissement des acteurs n'est qu'une conséquence acceptée tant que l'objectif est atteint. Ainsi était réglée de manière pragmatique la tension inévitable entre intérêts du roi et intérêts des financiers. Aguado était indispensable jusqu'en 1829, il le devint progressivement de moins en moins par la suite, à mesure que s'affirmait le caractère suicidaire de la politique dont il est l'instrument. L'ensemble de la dette émise permit de recueillir 715 millions de réaux, mais le total des intérêts et amortissements versés sur la même période s'élevait à 672,9 millions ${ }^{56}$. Le montant annuel des intérêts et amortissements à verser était passé de 16,7 millions de réaux en 1824 à 146,2 millions en $1833^{57}$, à un moment où les recettes de l'État ne dépassaient pas 450 à 500 millions de réaux. En d'autres termes, l'Espagne était arrivée à la situation absurde dans laquelle les emprunts nouveaux servaient à honorer le service de la dette. La chute d'Aguado en 1832 correspond donc à la disgrâce du ministre des Finances. L'épisode ressemble à la ${ }_{58}$ chute d'une équipe, telle qu'elle a pu survenir à plusieurs reprises au siècle précédent ${ }^{58}$. Toutefois, l'ampleur des déficits et la situation politique intérieure beaucoup plus complexe que par le passé, est aussi porteuse de ruptures.

\section{L'affaire des emprunts dans la politique globale de López Ballesteros}

Pour mieux être évaluée, l'affaire des emprunts et les acteurs qu'elle a mis en mouvement doivent être replacés dans le cadre de la politique économique globale menée par le pouvoir. L'ensemble des initiatives prises voit se dessiner un recul de l'État producteur hérité des Lumières dont tire profit un groupe composé en grande partie par ceux qui s'étaient enrichis à la faveur des emprunts. Le recul de l'État apparaît au travers du retour à la régie indirecte pour des activités qui étaient passées à la régie directe dans la deuxième moitié du XVIIIe siècle. Trois domaines sont touchés : les mines, les douanes et la fiscalité. Les établissements miniers avaient été détruits partiellement ou totalement pendant la guerre d'Indépendance. Faute de moyens pour remettre en état les installations, la paralysie s'installe. Une première initiative, limitée mais éclairante, est prise en 1817 : l'abolition du monopole d'État sur le plomb, avec toutefois des restrictions, et la relance de l'exportation de ce produit. Puis, la législation minière de 1825 réduit à huit les établissements de la Couronne et, faute de moyens, il est décidé d'affermer pour vingt ans les mines de la

\footnotetext{
${ }^{55}$ Le premier était procureur du conseil des finances à partir de 1829 , le second, depuis 1825 , secrétaire de la Trésorerie Générale et auteur d'un long rapport sur l'emprunt Guebhard. Suárez : 1970 b, vol. 1, p. 11.

${ }_{57}^{56}$ Broder : 1981, p. 445.

${ }^{57}$ Fontana : 2001, p. 167.

${ }^{58}$ Je renvoie ici aux articles de Carmen Sanz Ayán, Anne Dubet et Jean-Pierre Dedieu que 1'on trouvera dans ce livre.
} 
couronne (à l'exception d'Almadén) ${ }^{59}$.

La politique définie par López Ballesteros consistait à augmenter les recettes et réduire les dépenses. Côté dépenses, la principale initiative consistait à réduire le nombre des employés de l'État. Le ministère des finances était, de loin, le plus gros pourvoyeur d'emplois publics, c'est donc en son sein que furent effectuées les coupes les plus importantes. Le moyen le plus simple pour y parvenir était le retour à l'affermage de certains impôts, tel celui du «droit des portes ». Ce retour débuta en 1825 par le «droit des portes» de Grenade, puis de Séville et Cadix l'année suivante $^{60}$, avant d'être acquis par le banquier Felipe Riera pour l'ensemble du pays, à l'exception de Madrid, en 1830. L'État tenta aussi de revenir à l'affermage pour le tabac mais l'affaire échoua devant l'opposition des ultras et la peur du mécontentement populaire ${ }^{61}$. Toujours dans le but de réduire le nombre d'employés, la gestion du corps de la douane maritime (el resguardo marítimo) des Baléares fit l'objet d'une concession en $1829^{62}$.

Un autre volet de cette politique apparaît dans les initiatives prises pour relancer des projets de travaux publics entamés au XVIIIe siècle et abandonnés depuis. Le premier est la reprise de la construction du Canal de Castille qui devait relier Ségovie à Santander. Contre l'engagement à terminer le canal en sept ans, la cédule royale du 17 mars 1831 accordait la concession dans des conditions très favorables: exploitation de toutes les formes d'usage du canal pendant 80 ans, dotation annuelle de l'État de 300000 réaux, ainsi que du produit de l'impôt sur le vin de la province de Palencia pendant 25 ans. Dans les Asturies, une concession est accordée pour l'exploitation du petit gisement houiller d'Arnao. L'affaire débouche sur la fondation en avril 1834 de la Compagnie Royale des Asturies (Real Compañía Asturiana), moyennant 25 années d'exemption fiscale ${ }^{63}$. Le dernier chantier d'ampleur est celui de la mise en culture des îles du Guadalquivir à Séville. La principale d'entre elles, fut cédée en emphytéose en $1829^{64}$.

L'ensemble de ces initiatives repose sur la volonté de réduire les dépenses de l'État et de créer ou recréer des recettes nouvelles. Le court terme et le pragmatisme ont conduit à la mise en place de réformes techniques telles que l'élaboration des premiers budgets à partir de 1828, dans un souci de clarification et de contrôle. Toutefois, ces initiatives masquaient l'existence de deux conceptions opposées. Des secteurs du pouvoir étaient sensibles aux thèses économiques libérales. Des mesures dans ce sens furent prises sous le gouvernement libéral du Trienio, mais, parfois avec les même hommes, l'élan se poursuivit par la suite durant l'absolutisme restauré au travers de mesures phares comme la création du premier code de commerce en 1829 et de la Bourse de Madrid deux ans plus tard. Les partisans de l'État producteur étaient par ailleurs affaiblis par le discrédit qui touchait une part importante des techniciens issus de la tradition des Lumières, car ces hommes s'étaient très

\footnotetext{
${ }^{59}$ Chastagnaret : 2000, p. 122-124.

${ }^{60}$ Suárez : 1970b, vol. 1, p. 297.

${ }^{61}$ Fontana : 2001, p. 71.

${ }^{62}$ Actas del Consejo de Ministros, session du 21 février 1829.

${ }^{63}$ Ojeda : 1985, p. 14-15. Chastagnaret : 1989, p. 96-97.

${ }^{64}$ Moral Iruarte : 1990.
} 
majoritairement ralliés à l'Empire pendant la guerre d'Indépendance ${ }^{65}$. Toutefois, les hommes issus de cette tradition n'avaient pas désarmé. Ainsi, le maître à penser du texte de 1825 dans le secteur minier, Fausto de Elhuyar, restait culturellement un homme des Lumières et pensait l'affermage comme une parenthèse nécessaire liée à la conjoncture, l'État devant retrouver son rôle de producteur à l'issue des vingt années du contrat d'affermage ${ }^{66}$. Le paradoxe est de constater comment l'opposition ultra conservatrice s'était convertie au faire-valoir direct en dénonçant l'asiento. Arias Teijeiro notait dans son journal : «L'État ne pourrait-il pas gagner ce que gagnent les fermiers (rejetés par tous les économistes sains et judicieux) en ayant de bons employés $(. .$.$) ? { }^{67}$. Le très réactionnaire conseil d'État s'élevait contre l'affermage de l'impôt du droit des portes en affirmant que l'expérience « conseille d'arracher les recettes fiscales des mains des asentistas, libérant ainsi les peuples de la violence de leur cupidité ${ }^{68}$.

Une poignée d'hommes furent les bénéficiaires du retour à la régie indirecte. Se trouvent parmi eux plusieurs acteurs des emprunts : Aguado pendant un temps limité (pour le canal de Castille, les îles du Guadalquivir et les mines de plomb de la Sierra de Gador ${ }^{69}$ ), Casa Irujo et Burgos (canal de Castille et îles du Guadalquivir). Un autre ancien afrancesado, Luis de Figueroa, profita très largement de la conjoncture dans le commerce et la transformation du plomb. Il montra au pouvoir espagnol qu'un marché pouvait s'ouvrir à Marseille pour le plomb andalou. En 1822, puis 1823, deux contrats lui confièrent l'écoulement de 300000 quintaux de minerai des gisements andalous. Il loua aussi en 1825 pour dix ans la plus grande fonderie de la Couronne, dans la Sierra de Gador. À partir de 1822, le négociant marseillais paya un prix dérisoire à l'État et le minerai, malgré le coût du transport jusqu'à Malaga, parvenait dans ce port «à un prix inférieur de $40 \%$ environ au cours du marché sur la côte méditerranéenne $»^{70}$. C'est ainsi que Figueroa engrangea très rapidement des profits considérables. Les autres principaux bénéficiaires du retour à la régie indirecte furent deux banquiers : Felipe Riera (affermage du "droit des portes », des douanes des Baléares, des mines de charbon des Asturies) et Gaspar de Remisa (concession de l'exploitation des mines de Guadalcanal, de Río Tinto et du canal de Castille, traitement des scories et déblais divers du minerai de plomb dans la Sierra de Gador). Ces deux hommes avaient un passé de munitionnaires aux armées et restaient parmi les principaux munitionnaires à partir de 1823. Remisa avait obtenu d'Ouvrard, dans le cadre des célèbres contrats signés par le banquier avec l'armée française, l'approvisionnement des troupes françaises en Catalogne et au Levant lors de l'invasion des Cent Mille fils de Saint-Louis en $1823^{71}$. Il devint ensuite le fournisseur des garnisons françaises de Catalogne et du Levant restées en Espagne jusqu'en 1828. Il ne s'agit pas là de contrats passés directement avec l'État espagnol, mais l'identité du bénéficiaire avait forcément l'aval de la puissance publique ${ }^{72}$. Felipe Riera obtint à partir de 1823 l'approvisionnement particulièrement juteux de la marine à Cadix, pour

\footnotetext{
${ }^{65}$ Dans le secteur minier : Chastagnaret : 2000, p. 120.

${ }^{66}$ Chastagnaret : 1993b.

${ }^{67}$ Arias Teijeiro : 1967-1968, t. 2, p. 78.

${ }^{68}$ Suárez : 1970b, vol. 1, p. 297.

${ }^{69}$ Dans les trois cas, il renonça au bout de quelques mois. Luis : 2009, p. 233-239.

${ }^{70}$ Chastagnaret : 2000, p. 181-182. Chastagnaret : 1993a.

${ }^{71}$ Arias Teijeiro : 1967-1968, t. 1, p. 23, n. 44.

${ }^{72}$ Ramón de San Pedro : 1953, p. 38.
} 
une valeur s'élevant à 10 millions de réaux ${ }^{73}$.

En outre Casa Irujo, Remisa et Riera étaient directement ou indirectement intégrés dans l'appareil d'État. Le premier fut à plusieurs reprises ambassadeur, Riera devint trésorier des Finances des infants Francisco de Paula et Luisa Carlotta et Remisa membre du Conseil des Finances. Ce dernier accéda aussi à la prestigieuse fonction de directeur du Trésor royal en août 1826, dotée d'un salaire annuel de 100000 réaux, l'un des plus élevés de la fonction publique. Il conserva ce poste jusqu'à la fin de l'année 1833, tout en poursuivant ses activités de banquier ${ }^{74}$.

A ces deux hommes s'ajoute Manuel Gaviria y Romero. Ce sévillan devenu négociant à Madrid après la guerre d'Indépendance était parvenu à se glisser parmi les intimes de la famille royale durant les dernières années du règne de Ferdinand VII et était célèbre pour ses troupeaux de toros bravos et pour le titre de directeur du troupeau royal octroyé par le roi en 1831. Il était munitionnaire des troupes suisses et fermier de l'impôt de 5\% pesant sur la production des mines de plomb de la province de Grenade en $1832^{75}$.

Selon un phénomène classique, ces financiers ont accumulé les honneurs pour récompenser les services rendus à l'État, c'est-à-dire pour avoir accepté de faire au Trésor Royal les avances prévues dans le cadre des contrats. Riera et Aguado sont faits marquis et, aux côtés de Remisa, Gaviria, Casa Irujo et Burgos, chevaliers de l'ordre de Charles III $^{76}$. Cette poignée d'hommes se retrouvent dans des opérations communes, par exemple Burgos, Remisa, Casa Irujo et Aguado dans la société du canal de Castille. Remisa s'est associé avec Figueroa en 1826 dans le cadre d'un contrat de quatre ans pour le traitement des scories et déblais divers des mines de la Sierra de Gador ${ }^{77}$. Riera et Erro se rendaient ensemble au théâtre ${ }^{78}$. Ainsi s'explique le développement du sentiment qu'un groupe étroit de financiers liés au ministère des finances contrôle l'État. L'ultra Arias Tejero le déplorait en 1829: «Malheureuse nation, devenue le patrimoine d'une demi-douzaine de coquins qui détiennent le monopole des théâtres, des élevages de taureaux, de chevaux, l'approvisionnement aux armées, les mines, les douanes maritimes et maintenant le droit des portes $»{ }^{79}$. La politique de López Ballesteros est ainsi parvenue à assurer provisoirement la survie de l'absolutisme, mais elle l'a fait à crédit et au prix du développement d'un affairisme qui choque dans un pays qui n'avait pas encore achevé sa reconstruction après le traumatisme de la guerre d'Indépendance et de la perte de l'Empire.

\footnotetext{
${ }^{73}$ Il obtenait à cet effet des remboursements mensuels de la Hacienda d'une valeur de 100000 réaux. AHN, Hacienda, libro 6146.

${ }^{74}$ Un exemple de ses conseils en placement en 1832 dans Ramón de San Pedro : 1953, p. 96-97.

${ }^{75}$ AHN, Hacienda, libro 6146 et Otazu : 1987, p. 471. Il avança au titre de cet affermage 900 000 réaux au Trésor Royal.

76 Respectivement marquis de Casa Riera en février 1834, et marquis de las marismas del Guadalquivir en 1830 .

${ }^{77}$ Chastagnaret : 2000, p. 189.

${ }^{78}$ Suárez : 1970b, vol. 1, p. 296.

${ }^{79}$ Arias Teijeiro : 1967-1968, t. 2, p. 78.
} 


\section{Conclusion}

Répétons-le : la rareté des travaux de fond limite fortement nos connaissances sur le détail des contrats entre les financiers et l'État. On ignore en particulier tout de la cascade des sous-traitants des grands contrats signés à Madrid. L'affaire des emprunts évoquée ici est exceptionnelle et n'a pu se développer qu'à la faveur de circonstances elles aussi exceptionnelles qui n'ont duré que quelques années. Toutefois, l'importance de l'épisode, la mise en valeur de ses différents acteurs et les relations établies avec d'autres contrats entre État et financiers permettent de proposer un certain nombre d'hypothèses.

1. Sous le règne de Ferdinand VII, l'État a désormais comme partenaire une nouvelle génération de financiers. Le réseau qui s'est constitué autour de López Ballesteros, tout comme ceux avec lesquels il se trouve en concurrence, ne comprend pas de grands entrepreneurs du règne de Charles IV. Il y eut bien sûr par le passé des ruptures fortes, des disgrâces brutales, mais elles n'atteignaient jamais l'impression de table rase qui prévaut après la guerre d'Indépendance ${ }^{80}$. La coupure semble même plus forte que celle qui a marqué la Guerre de Succession d'Espagne un siècle plus tôt. L'effondrement de l'État en 1808 a entraîné avec lui les familles de financiers qui étaient ses partenaires privilégiés. Les nouvelles familles de financiers que l'on voit émerger sont issues des oligarchies urbaines et découvrent (Figueroa, Burgos) ou retrouvent (Aguado) le négoce et la finance qu'elles avaient fait passer au second plan. D'autres appartenaient avant 1808 au monde du commerce à un niveau modeste (Remisa, Riera, Gaviria). La guerre et l'approvisionnement aux armées ont joué un rôle fondamental dans l'essor de cette génération, par exemple chez Riera et Remisa ${ }^{81}$. Les emprunts de la dernière décennie du règne de Ferdinand VII constituent un autre vecteur puissant d'enrichissement rapide. Tous les membres du groupe étudié dans cet article ne connaissent cependant pas la même fortune : les Carrese étaient avant 1808 probablement davantage intégrés que les Riera, Remisa ou Burgos dans les circuits financiers de la monarchie puisque le père de Joaquín était lié à la Compagnie Guipuzcoana de Caracas ${ }^{82}$. Après 1808, Joaquín, tout comme son frère Antonio, restent au niveau d'intermédiaires percevant commission sans accéder au premier plan ${ }^{83}$. Rupture et continuité caractérisent enfin l'origine géographique de la nouvelle génération qui émerge sous Ferdinand VII. La dynamique de la période antérieure à 1808 donne ses derniers effets avec une présence importante de Basconavarrais et de familles d'Andalous, souvent issus de la zone basco-navarraise. La nouveauté vient de l'entrée en force du négoce et de la banque catalane à la cour. Remisa est le témoin et l'acteur principal de ce phénomène qui prendra de l'ampleur sous Isabelle II.

2. Le mouvement qui avait marqué le dernier tiers du XVIIIe siècle, celui du renforcement de l'État face aux financiers, s'interrompt et s'inverse sous Ferdinand VII. En témoignent d'abord l'ampleur des fortunes réalisées rapidement et l'ambiance d'affairisme qui est dénoncé. En témoigne surtout l'épuisement du phénomène de l'entrée des familles de négociants-financiers dans la haute fonction

\footnotetext{
${ }^{80}$ González Enciso : 2008.

${ }^{81}$ Des détails sur l'ensemble des affaires de ces hommes dans Luis : 2011.

${ }^{82}$ Chaparro Saínz : 2009, p. 423.

${ }^{83}$ Sur le rôle d'Antonio Carrese : Luis : 2009, p. 168 et 202.
} 
publique. Ofalia, et surtout Encima y Piedra constituent les derniers exemples de ces familles qui ont privilégié le service de l'État, sans abandonner toutefois le négoce. Encore une fois, Remisa symbolise la nouveauté : les financiers devenus administrateurs ne font pas souche dans l'appareil d'État qui a perdu en grande partie son prestige et n'est plus une garantie de sécurité depuis la guerre d'Indépendance. Une fois abandonnée sa fonction de directeur du Trésor Royal, Remisa revient exclusivement à la direction de sa banque dès janvier 1834 .

L'inversion de la tendance observée avant 1808 vient de l'affaiblissement de l'État, de sa détresse financière et de la situation de blocage politique qui prévaut alors. Cette situation favorise l'émergence d'un groupe administrativo-financier qui sait se rendre indispensable tout en profitant de toutes les opportunités offertes par le service de l'État pour s'enrichir. L'État perd aussi de son poids à cause de la nécessité de trouver de l'argent frais à l'étranger. Il est contraint de s'adresser à des financiers disposant de contacts hors de la péninsule qui, pour cette même raison, sont moins dépendants à son égard. Sous Ferdinand VII, l'État espagnol commence ainsi à être fortement dépendant des capitaux étrangers.

Alors que l'État semble s'affaiblir, la corruption et l'affairisme au service de l'État sont dénoncés, ce qui constitue une nouveauté. Ce qui était légitime auparavant ${ }^{84}$ semble ainsi de moins en moins accepté. Ce terrain est largement en friche pour les deux premiers tiers du XIXe siècle ${ }^{85}$. On doit pour le moment se contenter d'observer ici un double phénomène. D'une part l'émergence d'une différenciation entre sphère du public et sphère du privé, en conformité avec le changement de paradigme politique, social et juridique connu par le monde occidental depuis la fin du XVIIIe siècle. D'autre part une manifestation indirecte de l'affaiblissement de l'État : certaines formes de corruption (par exemple les cadeaux) ou d'instrumentalisation étaient acceptées quand, en retour, la puissance publique offrait cohérence, débouchés pour les élites et protection. Ce n'était plus le cas sous Ferdinand VII : la légitimité de l'absolutisme n'était plus l'objet d'un consensus et l'État monarchique avait perdu la grande capacité de régulation sociale qu'il detenait auparavant.

3. Le groupe qui émerge sous Ferdinand VII survit au souverain. Il perd certes ses relais dans l'administration avec la mise à l'écart de l'équipe de López Ballesteros en 1834 et son remplacement par des fidèles du nouveau ministre des finances, le comte de Toreno, dans les premiers gouvernements du nouveau régime libéral fondé quelques mois après la mort de Ferdinand VII ${ }^{86}$. Aguado n'est plus le banquier de la cour, il perd ce rôle privilégié au profit des Rothschild. Le groupe perd définitivement ses ultras avec l'engagement d'Erro dans les rangs carlistes. Enfin, la concurrence avec d'autres groupes politico-financiers, celui du comte de Toreno ou celui qui s'est réuni autour du financier libéral "progressiste » Mendizábal, est forte. Toutefois, le groupe qui dominait à la fin du règne de Ferdinand VII intègre de nouveaux financiers tels O'Shea et conserve des atouts en entrant dans l'intimité de la régente MarieChristine par l'intermédiaire de Remisa et de Gaviria, Gaviria fils devenant le

\footnotetext{
${ }^{84}$ Voir les entrées « Fraude » et « Corruption », rédigées par Anne Dubet dans Legay : 2010.

${ }^{85}$ Une exception notable pour les Philippines : Huetz de Lemps : 2006.

${ }^{86}$ Luis : 2002, p. 215-217.
} 
puissant intendant du palais. Ils commencent alors à collaborer avec Fernando Muñoz, l'époux morganatique de la régente ${ }^{87}$. Enfin, ces hommes conservent des positions fortes parce qu'ils participent activement au financement de la guerre carliste.

Le groupe revient provisoirement au premier plan en 1838 quand Ofalia accède à la tête du gouvernement. Ce dernier envoie Remisa et Joaquín Carrese à Paris, avec l'aide de Burgos et de Miñano afin de préparer le retour d'Aguado dans les emprunts d'État espagnols et de lui permettre de soustraire aux Rothschild l'exploitation des mines de mercure d'Almadén. L'affaire échoue, mais Aguado reçoit en compensation la concession des mines des Asturies ${ }^{88}$.

Les liens se renforcent ensuite avec l'entourage de Marie-Christine lors de l'exil de cette dernière à Paris sous la régence d'Espartero (1840-1843). Les groupes politico-financiers qui s'affrontaient dans les années 1830 finissent alors par trouver un terrain d'entente en se partageant affaires et pouvoir politique au sein du parti libéral modéré. Ils appliquent ainsi le principe élaboré par Fernando Muñoz : l'unité d'un parti est liée à la solidité des intérêts matériels communs de ses membres ${ }^{89}$.

4. L'affaiblissement de l'État, la dépendance à l'égard des capitaux étrangers, l'accroissement du poids et de l'autonomie des financiers dans les affaires publiques sont autant d'éléments nouveaux qui apparaissent sous Ferdinand VII. Cette évolution est à relier à l'effondrement de la monarchie impériale absolue et donc d'un système politico-social global dont le roi était la clé de voûte car la faveur du roi et le service de l'État étaient indispensables dans la course aux honneurs et à la richesse. Cette place centrale et sacrée du roi est contestée sous Ferdinand VII, pas seulement par les libéraux, mais aussi par une partie des forces sociales vives qui se rendent compte que le roi n'a plus ni les moyens matériels ni la légitimité pour conserver la place éminente dont il disposait auparavant ${ }^{90}$. De manière très révélatrice, les familles de financiers n'entrent plus dans la haute fonction publique après 1834. Ils deviennent hommes politiques en briguant des sièges de sénateurs ou de députés et ne retrouvent l'appareil d'État qu'en accédant à des postes ministériels. La nature profonde de l'État et les sources de sa légitimité ont changé et avec elles les relations entre les élites sociales et le pouvoir politique suprême. Cette mutation n'attend pas la fin de l'absolutisme. Elle débute avec la guerre d'Indépendance qui, dans le domaine de la relation entre les financiers et l'État comme dans de nombreux autres domaines, marque bien l'effondrement d'un monde.

\footnotetext{
${ }^{87}$ Plus tard, en 1847, la fille de Remisa épouse Jesús Muñoz, le jeune frère de Fernando Muñoz.

${ }^{88}$ Luis : 2009, p. 250-254.

${ }^{89}$ Pro Ruiz : 2007, p. 32.

${ }^{90}$ Je renvoie pour une réflexion globale à Dedieu : 2010.
} 